\title{
RELAÇÃO ENTRE O ÍNDICE DE RESISTÊNCIA OBTIDO PELA ULTRA-SONOGRAFIA DOPPLER TRANSFONTANELA E O NEURODESENVOLVIMENTO ATÉ O PRIMEIRO ANO DE VIDA EM RECÉM-NASCIDOS A TERMO COM ENCEFALOPATIA HIPÓXICO-ISQUÊMICA LEVE E MODERADA
}

\author{
Maria Helena Martins Garcia', Alexandra Maria Vieira Monteiro², Sergio Miranda Freire ${ }^{3}$
}

\begin{abstract}
RESUMO - Objetivo: Avaliar a relação do índice de resistência (IR) obtido pela ultra-sonografia Doppler transfontanela com o neurodesenvolvimento até um ano de idade, em recém-nascidos (RN) a termo com encefalopatia hipóxica-isquêmica $(\mathrm{EHI})$ leve a moderada, secundária à asfixia intra-parto. Método: Estudo prospectivo em $20 \mathrm{RN}$ com EHI leve a moderada, IR elevado no primeiro exame de Doppler, e sem doenças associadas ou anormalidades morfológicas cerebrais. Foram realizados exames seriados bimensais de Doppler transfontanela a partir do sétimo dia de vida, e avaliações clínicas mensais do neurodesenvolvimento no primeiro ano de vida. Resultados: Houve normalização progressiva dos valores de IR até o último exame realizado. Cinco pacientes apresentaram normalização clínico-neurológica no período neonatal, após o primeiro exame de Doppler. Quinze lactentes apresentaram alterações neurológicas com resolução a partir do segundo trimestre de vida. Conclusão: Houve relação entre os períodos em que ocorreu a normalização dos valores de IR e a melhora clínica-neurológica.
\end{abstract}

PALAVRAS-CHAVE: encefalopatia hipóxica-isquêmica, ultra-sonografia Doppler, recém-nascido, neurodesenvolvimento.

\begin{abstract}
Relation between the resistance index obtained by the transfontanellar Doppler ultrasonography and the neurological development until the first year of life in term infants with mild or moderate hypoxic-ischaemic encephalopathy

ABSTRACT - Objective: To evaluate the relation between the resistance index (RI) obtained by transfontanellar Doppler ultrasonography, and the neurodevelopment until one year of life, at term newborns with mild or moderate hypoxic-ischaemic encephalopathy due to intrapartum asphyxia. Method: 20 term newborns, with mild or moderate hypoxic-ischemic encephalopathy, high values of resistance index in the first exam, and without cerebral morfologic abnormalities or other diseases. They were submitted to serial bimonthly transfontanellar Doppler ultrasonography, from the seventh day of life on, and monthly clinical neurodevelopment assessment until one year of life. Results: There was a progressive normalization of RI values until the last examination. In five cases there were clinical neurologic normalization in the neonatal period after the first Doppler exam. Fifteen infants presented neurologic abnormalities, with normalization after the second trimester of life. Conclusion: There was a relation between the normal RI values with the normalization of the clinical assessment.
\end{abstract}

KEY WORDS: hypoxic-ischaemic encephalopathy, Doppler ultrasonography, newborn, neurodevelopment.

\begin{abstract}
Departamento de Pediatria e Unidade Docente-Assistencial de Radiologia (UDA) do Hospital Universitário Pedro Ernesto (HUPE). Programa de Pós-graduação em Ciências Médicas (PGCM), Universidade do Estado do Rio de Janeiro, Rio de Janeiro RJ, Brasil: 'Pósgraduanda do Programa de Pós-graduação em Ciências Médicas da Faculdade de Ciências Médicas (FCM-UERJ), Médica neonatologista do Hospital Universitário Pedro Ernesto (HUPE-UERJ); ${ }^{2}$ Professor Adjunto (Doutor em Medicina) de Radiologia dos Cursos de Graduação e Pós-graduação da Faculdade de Ciências Médicas da Universidade do Estado do Rio de Janeiro (FCM-UERJ); ${ }^{3}$ Professor Adjunto do Departamento de Tecnologia da Informação e Educação em Saúde, da Faculdade de Ciências Médicas da Universidade do Estado do Rio de Janeiro (FCM-UERJ).
\end{abstract}

Recebido 8 Maio 2007, recebido na forma final 22 Agsoto 2007. Aceito 25 Setembro 2007.

Dra. Maria Helena Martins Garcia - Rua Dona Delfina 28 / 801 - 20511-270 Rio de Janeiro RJ - Brasil. E-mail: mhelenamg@terra.com.br 
A asfixia intra-parto com lesão cerebral hipóxicaisquêmica é o principal fator de morbidade a longo prazo em recém-nascidos (RN) a termo', com incidência mundial de dois a quatro para cada 1000 nascimentos a termo, segundo a Organização Mundial de $S a^{2} e^{2}$. As seqüelas mais freqüentes da encefalopatia hipóxica-isquêmica (EHI) são as alterações de tônus muscular e os danos motores, sendo a encefalopatia crônica não progressiva a expressão mais grave de dano motor ${ }^{3}$. Podem ocorrer também alterações visuais, déficit auditivo neuro-sensorial, e déficit cognitivo. Ainda permanece difícil predizer quais RN terão seqüelas neurológicas a longo prazo; é necessário, portanto, ter um marcador útil para o prognóstico neurológico, pois estratégias promissoras de intervenção em reabilitação são disponíveis atualmente ${ }^{4}$.

Neste contexto, os métodos de imagem têm sido utilizados nas avaliações das alterações morfológicas no sistema nervoso central (SNC) e das modificações no fluxo sangüíneo cerebral (FSC) ${ }^{5,6}$. Em relação ao FSC de RN, os mecanismos de auto-regulação vascular cerebral do RN a termo são mais amadurecidos em relação ao prematuro, porém sensíveis a condições de hipóxia. O índice de resistência (IR), aferido pela ultrasonografia transfontanela com Doppler, traduz as alterações no FSC provocadas pela hipóxia7, tendo sido utilizado neste grupo de pacientes por ser um método de imagem não invasivo, que não utiliza radiação ionizante, de fácil reprodução e de fácil transporte do aparelho, além de apresentar concordância inter-observador satisfatória ${ }^{8-10}$.

O objetivo deste estudo foi verificar, em RN a termo com diagnóstico de $\mathrm{EHI}$ leve ou moderada por asfixia intra-parto, com ultra-sonografia transfontanela morfológica normal e sem doenças associadas, a relação entre a elevação do IR, aferido pelo Doppler transfontanela, e o neurodesenvolvimento até o primeiro ano de vida.

\section{MÉTODO}

Foram incluídos no estudo $20 \mathrm{RN}$ a termo, nove do sexo feminino e onze do sexo masculino, com média de idade gestacional de 39 semanas e um dia (DP-desvio padrão- 9,5 dias) e média de peso de nascimento 3080 gramas, (DP$0,462 \mathrm{~g}$ ), internados na Unidade de Terapia Intensiva Neonatal do Hospital Universitário Pedro Ernesto da Universidade do Estado do Rio de Janeiro, no período de setembro de 2003 a julho de 2005. Todos apresentaram diagnóstico de EHI leve ou moderada secundária a asfixia intra-parto, USTF morfológica normal e IR elevado no primeiro exame de Doppler. Para o diagnóstico de asfixia intra-parto, foi considerada a presença de no mínimo três dos seguintes critérios $^{11}$ : anormalidades na cardiotocografia fetal, presença de líquido amniótico meconial, evento agudo obstétrico, índi- ce de Apgar igual ou menor a seis no quinto minuto de vida, acidose metabólica em amostra de sangue arterial do RN na primeira hora de vida, e evidência precoce de comprometimento sistêmico (miocárdico, renal e intestinal). Para a classificação da EHI, foram utilizados os critérios de Sarnat e Sarnat ${ }^{12}$ para leve, moderada e grave; só foram incluídos os casos diagnosticados como leve e moderada. Todos os RN diagnosticados como EHI grave foram excluídos por razões diversas tais como: dano cerebral estrutural, infecção neonatal, retardo do crescimento intra-uterino, e anomalias congênitas, dentre outras. Avaliações do IR foram obtidas até o sétimo mês de vida com intervalos para o primeiro exame, entre o sétimo e décimo-quarto dia de vida; segundo exame, entre o segundo e o terceiro mês de vida; terceiro exame, entre o quinto e sexto mês de vida e; quarto exame, entre o sexto e o sétimo mês de vida. Em 13 casos, não foi realizada a última avaliação devido à normalização dos valores de IR no exame anterior. Todos as avaliações foram realizadas pela fontanela anterior, em cortes coronais, nas artérias cerebrais média direita (ACMD) e esquerda (AC$M E)$, e em cortes sagitais para as artérias cerebral anterior (ACA) e artéria basilar ( $\mathrm{AB}$ ). $\mathrm{O}$ aparelho de ultra-sonografia utilizado foi da marca Toshiba, modelo Power Vision 6000, com transdutor convexo com freqüência de $3.5 \mathrm{MHz}$ para o Doppler. Todos os exames foram realizados por dois observadores, sendo que o IR foi selecionado e determinado automaticamente pelo aparelho de ultra-sonografia. Foram considerados alterados os valores de IR iguais ou maiores que 0,726 com desvio padrão de $0,057^{13}$. Após a alta dos RN da Unidade de Tratamento Intensivo Neonatal, avaliações clínicas mensais foram realizadas até um ano de idade pelo método de avaliação neurológica de Amiel-Tison ${ }^{14}$ e pelo Teste de Desenvolvimento de Denver $1 I^{15}$.

O estudo foi aprovado pelo Comitê de Ética em Pesquisa do Hospital Universitário Pedro Ernesto da Universidade do Estado do Rio de Janeiro, e o consentimento informado foi obtido de todos os responsáveis pelos pacientes.

Os dados obtidos neste estudo foram analisados estatisticamente pelo teste $t$ de Student pareado, para comparação das médias dos valores de índice de resistência encontrados em cada uma das artérias avaliadas, no primeiro e no terceiro exame. O nível de significância (valor alfa), escolhido para o teste $t$ foi igual a 0,05 .

\section{RESULTADOS}

Dentre os 20 RN incluídos no estudo, 13 RN apresentaram EHI leve, e sete RN apresentaram EHI moderada.

Em relação ao seguimento do $I R$, dos $20 \mathrm{RN}$, todos fizeram o segundo exame, 18 fizeram o terceiro exame, e somente sete lactentes realizaram quatro exames. Houve um lactente com IR anormal no terceiro exame que não pôde ser submetido ao quarto exame devido ao pequeno orifício da fontanela anterior.

$\mathrm{Na}$ Tabela 1, encontram-se a média, a mediana e o desvio-padrão dos valores de IR observados nos quatro exames seriados nas artérias basilar, cerebral ante- 
Tabela 1. Médias e medianas de índice de resistência nas artérias cerebrais.

\begin{tabular}{|c|c|c|c|c|}
\hline & \multicolumn{4}{|c|}{ IR } \\
\hline & $1^{\circ}$ exame & $2^{\circ}$ exame & $3^{\circ}$ exame & $4^{\circ}$ exame \\
\hline Média AB & 0,792 & 0,714 & 0,671 & 0,611 \\
\hline Mediana AB & 0,79 & 0,7 & 0,66 & 0,61 \\
\hline$D P A B$ & 0,053 & 0,079 & 0,078 & 0,056 \\
\hline Média ACA & 0,804 & 0,738 & 0,677 & 0,608 \\
\hline Mediana ACA & 0,8 & 0,725 & 0,675 & 0,58 \\
\hline DP ACA & 0,039 & 0,086 & 0,084 & 0,065 \\
\hline Média ACMD & 0,805 & 0,764 & 0,701 & 0,627 \\
\hline Mediana ACMD & 0,815 & 0,765 & 0,705 & 0,61 \\
\hline DP ACMD & 0,055 & 0,078 & 0,052 & 0,069 \\
\hline Média ACME & 0,806 & 0,754 & 0,725 & 0,655 \\
\hline Mediana ACME & 0,805 & 0,755 & 0,71 & 0,65 \\
\hline DP ACME & 0,055 & 0,075 & 0,062 & 0,061 \\
\hline
\end{tabular}

$I R$, índice de resistência; $A B$, artéria basilar; $A C A$, artéria cerebral anterior; $A C M D$, artéria cerebral média direita; ACME, artéria cerebral média esquerda; DP, desvio-padrão.

Tabela 2. Teste $t$ para diferença de médias entre $01^{\circ}$ e $03^{\circ}$ exame.

\begin{tabular}{lcccc}
\hline & $A B$ & $A C A$ & $A C M D$ & ACME \\
\hline Diferença de médias (IR) & 0,13 & 0,12 & 0,1 & 0,08 \\
IC $95 \%$ & 0,1 a 0,17 & 0,08 a 0,16 & 0,07 a 0,13 & 0,05 a 0,11 \\
Valor $p$ & 0,0000001 & 0,000001 & 0,000003 & 0,000044 \\
\hline
\end{tabular}

$I R$, índice de resistência; $A B$, artéria basilar; $A C A$ artéria cerebral anterior; $A C M D$, artéria cerebral média direita; $A C M E$, artéria cerebral média esquerda; IC $95 \%$, intervalo de confiança de $95 \%$.

Tabela 3. Alterações clínicas neurológicas nos períodos de exame de Doppler.

\begin{tabular}{lccc}
\hline & $1^{\circ}$ exame & $2^{\circ}$ exame & $3^{\circ}$ exame \\
\hline Amiel-Tison anormal & & & \\
Número de crianças & 15 & 6 & 3 \\
Porcentagem & $75 \%$ & $30 \%$ & $15 \%$ \\
IC $95 \%$ & $50,9 \%$ a $91,3 \%$ & $11,9 \%$ a $54,3 \%$ & $3,4 \%$ a $39,6 \%$ \\
Denver II anormal & & & \\
Número de crianças & 7 & 2 & 0 \\
Porcentagem & $35 \%$ & $10 \%$ & \\
IC $95 \%$ & $15,4 \%$ a $59,2 \%$ & $1,2 \%$ a $31,7 \%$ & 20 \\
Total de crianças & 20 & 20 & \\
\hline
\end{tabular}

IC $95 \%$, intervalo de confiança de $95 \%$.

rior, cerebral média direita, e cerebral média esquerda nos 20 pacientes. A Figura 1 representa o comportamento dos valores de IR em cada paciente, ao longo do tempo, na artéria basilar; nas outras três artérias o comportamento dos valores de IR foi semelhante. Foi observada uma diminuição dos valores de IR em relação ao tempo pelo teste t de Student. Nas quatro artérias analisadas, houve diferença estatisticamente significativa entre os valores de IR do primeiro e do terceiro exame, com valor $\mathrm{p}$ menor que 0,0001, como demonstrado na Tabela 2.

Em relação à avaliação do neurodesenvolvimento, a Tabela 3 mostra o número e o percentual de lactentes com alterações na avaliação de Amiel-Tison, e com atraso no desenvolvimento pelo teste de Denver II, para cada um dos três primeiros exames de Doppler. Segundo a avaliação de Amiel-Tison, 15 lactentes foram classificados como portadores de anormali- 


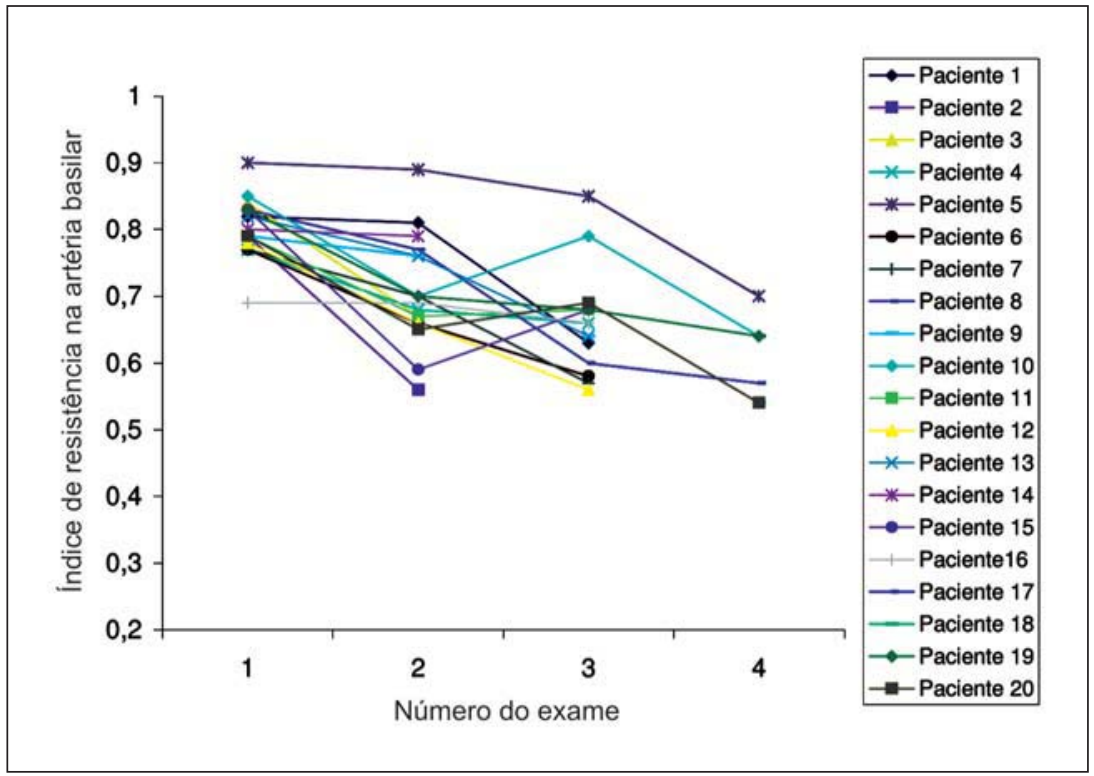

Fig 1. Valores de índice de resistência na artéria basilar.

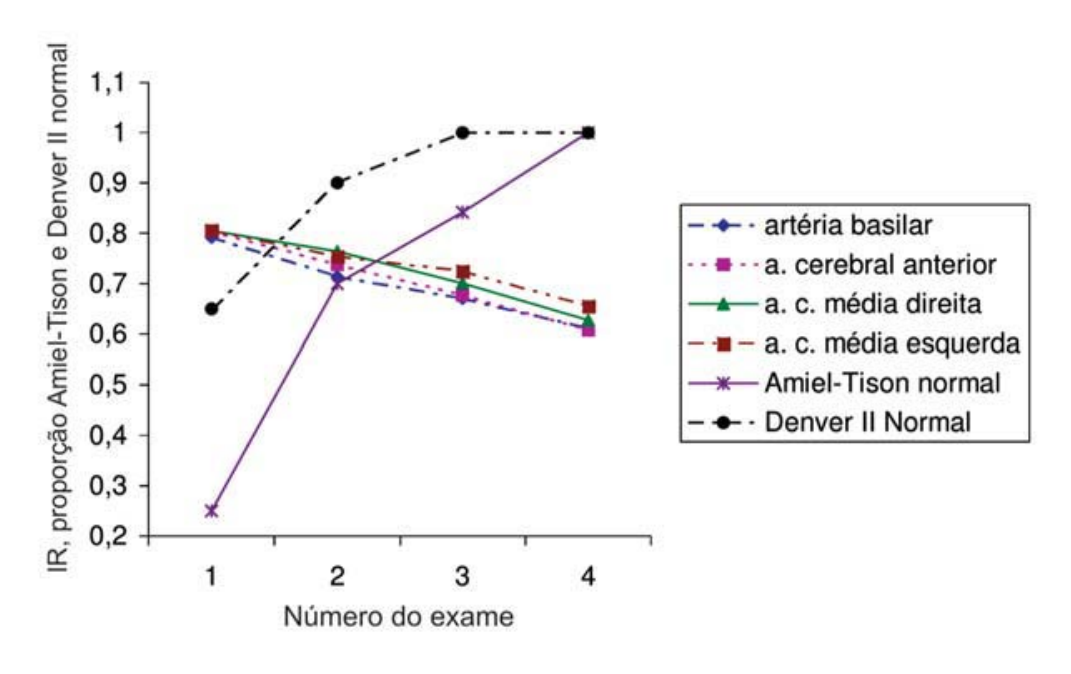

Fig 2. Médias de índice de resistência nas artérias cerebrais nos quatro exames, e a proporção de lactentes com avaliações clínicas neurológicas normais. a. cerebral anterior, artéria cerebral anterior; a.c. média direita, artéria cerebral média direita; a.c. média esquerda, artéria cerebral média esquerda.

dades transitórias, nove destas com normalização ao final do primeiro trimestre (época do segundo exame de Doppler), três com normalização no segundo trimestre (época do terceiro exame de Doppler), e três com normalização no terceiro trimestre (época de realização do quarto exame de Doppler). Segundo o teste de Denver II, sete lactentes apresentaram atraso no desenvolvimento no primeiro trimestre (estes também apresentaram alterações na avaliação de Amiel-Tison no mesmo período); no segundo trimestre, somente dois destes lactentes ainda apresentaram sinais de atraso no desenvolvimento; no terceiro trimestre todos os lactentes apresentaram desenvolvimento adequado para a idade.

A Figura 2 ilustra a comparação no decorrer do tempo entre a proporção de lactentes com avaliações de Amiel-Tison e de Denver II normais, e os valores das médias de IR nas quatro artérias analisadas. Nota-se no Gráfico que à medida que os valores de médias de IR diminuem no segundo, terceiro e quarto exames, a proporção de lactentes com avaliações clínicas normais aumenta.

\section{DISCUSSÃO}

Vários estudos sobre a dopplerfluxometria pela ultra-sonografia transfontanela em recém-nascidos têm sido realizados, demonstrando um declínio fisiológico progressivo do IR com a idade cronológica ${ }^{16}$, e alterações dos valores de IR em condições patológicas, como por exemplo na hipóxia ${ }^{17-25}$. Porém este pare- 
ce ser o primeiro estudo utilizando isoladamente o IR como possível exame de seguimento, no primeiro ano de vida, para o prognóstico neurológico em RN a termo com EHI leve ou moderada e com USTF morfológica normal.

Em estudos correlatos utilizando o Doppler transfontanela para a avaliação da EHI em RN a termo, não foram excluídos RN com alterações morfológicas detectadas pela USTF ${ }^{17-23}$. Observaram diminuição dos valores de IR em RN com EHI grave ou modera$\mathrm{da}$, associando os valores menores ou iguais a 0,55 , no período de seis horas a cinco dias de vida, a um pior prognóstico como morte no período neonatal e disfunção neurológica grave a longo prazo. Outros estudos incluíram RN com outras enfermidades além da asfixia intra-parto ${ }^{24,25}$.

Dos 20 RN incluídos neste estudo, 15 apresentaram anormalidades transitórias na avaliação neurológica e no desenvolvimento, com normalização a partir do final do segundo trimestre de vida. A evolução do neurodesenvolvimento no final do primeiro ano de vida foi adequada para a idade em todos os pacientes, o que está de acordo com outros relatos na literatura que destacaram a boa evolução dos casos de EHI leve e moderada em países desenvolvidos ${ }^{2,26}$, no entanto sem a correlação com um exame de avaliação vascular como o Doppler.

Neste estudo pôde-se determinar graficamente a relação entre o comportamento vascular arterial cerebral e o neurodesenvolvimento, e foi observado o declínio dos valores de médias de IR ao longo do tempo, associado à normalização da avaliação clínica. Houve concordância, em relação ao tempo, entre os comportamentos do IR e do neurodesenvolvimento até o primeiro ano de vida em RN com EHI leve a moderada. O seguimento deste grupo de pacientes será necessário para avaliação de alterações mais sutis a longo prazo.

Não foi possível ainda se estabelecer uma relação estatisticamente significativa entre o IR e o neurodesenvolvimento, devido ao número reduzido de pacientes, em conseqüência aos rigorosos critérios de inclusão. Estudos posteriores serão necessários para a definição do valor preditivo do Doppler transfontanela no prognóstico de RN a termo com asfixia intraparto e EHI.

\section{REFERÊNCIAS}

1. Simon NP. Long term neurodevelopmental outcome of asphyxiated newborns. Clin Perinatol 1999;26:767-778.

2. World Health Organization. World Health Report 2005. Newborns: no longer going unnoticed. Geneva. World Health Organization,2005 [Online]. http://www.who.int/whr/2005/chap5.

3. Hagberg B, Hagberg G, Beckung E, Uvebrant P. The changing panorama of cerebral palsy in Sweden. VIII. Prevalence and origin in the birth year period 1991-1994. Acta Paediatr Scand 2001;90:271-277.

4. Dennery PA. Predicting neonatal brain injury: are we there yet? Arch Pediatr Adolesc Med 2003;157:1151-1152.

5. Assis MC, Machado HR. Medida da velocidade de fluxo nas artérias cerebrais utilizando ultra-som Doppler transfontanela antes e após o tratamento cirúrgico da hidrocefalia. Arq Neuropsiquiatr 1999;57:827-835.

6. Muniz IACC, Netto AA, Gonçalves VMG. Velocimetria Doppler no período neonatal em recém-nascidos a termo pequenos para a idade gestacional. Arq Neuropsiquiatr 2003;61:808-815.

7. Volpe JJ. Hypoxic-isquemic encephalopathy. In: Neurology of newborn. 4.Ed. Philadelphia: WB Saunders, 2000:253;371-372.

8. Moorthy B, Colditz PR, Rees DG, et al. Reproducibility of cerebral artery Doppler measurements. Arch Dis Child 1990;65:700-701.

9. Ohlsson A, Fong K, Ryan ML, et al. Cerebral blood flow velocity measurements in neonates: technique and interobserver reliability. Pediatr Radiol 1991;21:395-397.

10. Volpe JJ, Perlman JM, Hill A, et al. Cerebral blood flow velocity in human newborn: the value of this determination. Pediatrics 1992;70:147-152.

11. Cowan F. Outcome after intrapartum asphyxia in term infants. Semin Neonatol 2000;5:127-140.

12. Sarnat HB, Sarnat MS. Neonatal encephalopathy following fetal distress: a clinical and electroencephalografic study. Arch Neurol 1976;33:696-705.

13. Allison JW, Faddis LA, Kinder DL, Robertson PK, Glasier CM, Seibert $\mathrm{JJ}$. Intracranial resistive index (RI) values in normal term infants during the first day of life. Pediatr Radiol 2000;30:618-620.

14. Amiel-Tison C. A method for neurologic evaluation within the first year of life. Curr Probl Pediatr 1976;7:1-50.

15. Frankenburg WK, Dodds J, Archer P, Shapiro H, Bresnick B. The Denver II: a major revision and retandardization of the denver developmental screening test. Pediatrics 1992;89:91-97.

16. Bode H, Wais U. Age dependence of flow velocities in basal cerebral arteries. Arch Dis Child 1988;63:606-611.

17. Archer LNJ, Levene MI, Evans DH. Cerebral artery Doppler ultrasonography for prediction of outcome after perinatal asphyxia. Lancet 1986:8516:1116-1118.

18. Levene MI, Fenton AC, Evans DH, Archer LNJ, Shortland DB, Gibson NA. Severe birth asphyxia and abnormal cerebral blood-flow velocity. Dev Med Child Neurol 1989;31:427-434.

19. Gray, Tudehope DI, Masel JP, et al. Perinatal hipoxic-ischaemic brain injury: prediction of outcome. Dev Med Child Neurol 1993;35:965-973.

20. Eken P, Toet MC, Groenendaal F, De Vries LS. Predictive value of early neuroimaging, pulsed Doppler and neurophysiology in full term infants with hypoxic-ischaemic encephalopathy. Arch Dis Child 1995;73:75-80.

21. Ilves P, Talvik R, Talvik T. Changes in Doppler ultrasonography in asphyxiated term infants with hypoxic-ischaemic encephalopathy. Acta Paediatr 1998;87:680-684.

22. Jongeling BR, Badawi N, Kurinczuk JL, et al. Cranial ultrasound as a predictor of outcome in term newborn encephalopathy. Pediatr Neurol 2002;26:37-42.

23. Ilves $P$, Lintrop M, Metsatht T, Vaher U, Talvik T. Cerebral blood-flow velocities in predicting outcome of asphyxiated newborn infants. Acta Paediatr 2004;93:523-528.

24. Stark JE, Seibert JJ. Cerebral artery doppler ultrasonography for prediction of outcome after perinatal asphyxia. J Ultrasound Med 1994;13:595- 600.

25. Fukuda S, Kato T, Kuwafara S, Kato I, Futamura M, Togari H. The ratio of flow velocities in the middle cerebral and internal carotid arteries for the prediction of cerebral palsy in term neonates. J Ultrasound Med 2005;24:149-153.

26. Ellis M, Manandhar N, Shrestha PS, Shrestha L, Manandhar DS, Costello AM. Outcome at 1 year of neonatal encephalopathy in Kathmandu, Nepal. Dev Med Child Neurol 1999;41:685-689. 